\title{
Strategy for salvaging infected breast implants: lessons from the recovery of seven consecutive patients
}

\author{
Hyeonjung Yeo ${ }^{1}$, Dongkyu Lee ${ }^{1}$, Jin Soo Kim², Pil Seon Eo ${ }^{2}$, Dong Kyu Kim², Joon Seok Lee², \\ Ki Tae Kwon ${ }^{3}$, Jeeyeon Lee ${ }^{4}$, Ho Yong Park ${ }^{4}$, Jung Dug Yang ${ }^{2}$ \\ ${ }^{1}$ Department of Plastic and Reconstructive Surgery, Daegu Fatima Hospital, Daegu; ${ }^{2}$ Department of Plastic and Reconstructive Surgery, \\ ${ }^{3}$ Division of Infectious Diseases, Department of Internal Medicine, and ${ }^{4}$ Department of Surgery, School of Medicine, Kyungpook National \\ University, Daegu, Korea
}

\begin{abstract}
Background In recent years, implant-based breast reconstruction has been performed because of its simplicity, short operation time, and rapid recovery of patients. Several studies have reported treatment methods for implant surgery-related infection, which is a serious complication. The aim of this study was to introduce our strategy for salvaging infected implants and to evaluate its effectiveness.

Methods The authors performed a retrospective study of 145 cases from 132 patients who underwent implant-based breast reconstruction from January 2012 to December 2018. Empirical antibiotics were immediately administered to patients with suspected infections. The patients then underwent salvage treatment including appropriate antibiotics, ultrasonography-guided aspiration, debridement, antibiotic lavage, and implant exchange through a multidisciplinary approach. Patient demographics, operative data, duration until drain removal, adjuvant treatment, and complications were analyzed.

Results The total infection rate was 5.5\% (8/145). A longer indwelling catheter period and adjuvant treatment were significantly associated with infection. The salvage treatment showed a success rate of $87.5 \%(7 / 8)$. Seven patients who received early aggressive salvage treatment recovered from infection. One patient with methicillin-resistant Staphylococcus aureus, who received salvage treatment 11 days after symptom onset, did not respond to drainage and antibiotic treatment. That patient subsequently underwent explantation.

Conclusions In implant-based breast reconstruction, prevention of infection is of the utmost importance. However, if an infection is suspected, proactive empirical antibiotic therapy and collaboration with the necessary departments are required. Through a multidisciplinary approach and proactive early management, swift and appropriate salvage should be performed.
\end{abstract}

Keywords Mammaplasty / Breast implants / Infections / Salvage therapy / Interdisciplinary studies
Correspondence: Jung Dug Yang Department of Plastic and Reconstructive Surgery, School of Medicine, Kyungpook National University, 130 Dongdeok-ro, Junggu, Daegu 41944, Korea Tel: +82-53-200-5681 Fax: +82-53-425-3879

E-mail: lambyang@knu.ac.kr

Received: August 10, 2020 • Revised: December 13, 2020 • Accepted: December 13, 2020

pISSN: 2234-6163 • elSSN: 2234-6171・https://doi.org/10.5999/aps.2020.01578• Arch Plast Surg 2021;48:165-174

Copyright $\odot 2021$ The Korean Society of Plastic and Reconstructive Surgeons

This is an Open Access article distributed under the terms of the Creative Commons Attribution Non-Commercial License (https://creativecommons.org/

licenses/by-nc/4.0/) which permits unrestricted non-commercial use, distribution, and reproduction in any medium, provided the original work is properly cited. I www.e-aps.org 


\section{INTRODUCTION}

According to the American Society of Plastic Surgeons, implantbased breast surgery accounted for the largest proportion of breast operations performed in the United States in 2018 [1]. Implant-based breast reconstruction is now considered the first choice for breast reconstruction because of its advantages, which include a short operation time due to the simplicity of the procedure, a rapid postoperative recovery, no donor site, and little scarring; however, its potential complications need to be further elucidated $[2,3]$. According to Cordeiro et al., the most common early complication of implantation is infection (2.5\%); other possible complications include capsular contracture, inaccurate positioning of implants, hematoma, and seroma. Peripheral inflammation of the skin flap and implant exposure due to necrosis are the principal serious complications [4-6].

Infection occurs more frequently after breast reconstruction than after breast augmentation. Although risk factors for postimplantation infection have not been investigated through longterm prospective studies, surgical techniques and patients' underlying diseases have been reported to be the most critical determinants. Mastectomy and radiotherapy are high-risk factors for infection. Though it is difficult to identify the source of infection in patients who undergo implantation, potential causes include contaminated implants or saline, surgical procedures or environment, and patients' skin tissue or mammary glands, as well as the possible spread of infection from distant areas. Approximately $60 \%$ of peri-implant infections occur immediately after surgery, whereas delayed infections can occur due to secondary bacteremia or invasive procedures on body parts other than the breast $[7,8]$. Leyngold et al. [9] reported that cellulitis, hospitalization (compared to outpatient treatment), wound dehiscence, and necrosis were major risk factors for infection after breast reconstruction using tissue expanders.

As breast reconstruction using implants and acellular dermal matrix $(\mathrm{ADM})$ without autologous tissue replacement has been widely performed, the management of implant-related infection, a potentially serious complication, has become an emerging issue. Infections after implant-based breast reconstruction can lead to hospitalization, delays in scheduled chemotherapy or radiotherapy, breast deformation due to implant failure, sepsis, or death. Although prevention is of the utmost importance for avoiding these complications, if infection does occur, the choice of subsequent treatment modalities may determine the patient's prognosis. For cases of infection after implant-based breast reconstruction, delayed reconstruction after implant removal and infection control has been the standard practice. However, these procedures complicate the subsequent reim- plantation and cannot guarantee esthetic results.

Recent studies have reported that active salvage treatment in the early stage was effective for peri-implant infection. In 2007, Chun et al. [10] stated that rapid and aggressive surgical intervention can salvage infected breast implants, which were previously considered impossible to save. A study by Prince et al. in 2012 [11] reported a salvage rate of $76.7 \%$ in patients with implant infection or exposure who were treated by rapid and aggressive surgical interventions with systemic antibiotics. Thus, we herein report a case series of infections after implant-based breast reconstruction that were treated successfully through a multidisciplinary diagnosis and early aggressive treatment.

\section{METHODS}

A retrospective chart review was performed on 145 cases in 132 patients who underwent implant-based breast reconstruction at two breast reconstruction centers between January 2012 and December 2018. The Institutional Review Board of Kyungpook National University Chilgok Hospital (IRB No. KNUCH 202007-019) approved the protocol of this study. The patients provided written informed consent for the publication and the use of their images.

\section{Patient demographics}

Data on the following known risk factors for postoperative infection were investigated: age, body mass index (BMI), underlying diseases, a history of chemotherapy or radiotherapy, and smoking history. In addition, medical records regarding axillary lymph node dissection, the use of ADM, the duration of postoperative drainage, symptoms of infection, culture results, treatment methods and duration, type of antibiotics administered, other accompanying complications, and follow-up period were collected.

\section{Surgical procedure}

Directly after mastectomy for breast cancer, breast reconstruction was performed in one stage through direct-to-implant breast reconstruction. Prepectoral and subpectoral pockets were defined according to the location of implant insertion. In prepectoral pockets, the implant was surrounded by crossing two $\mathrm{ADMs}$ and inserted onto the anterior surface of the pectoralis major [12]. Subsequently, the ADMs were fixed to the pectoralis major using Vicryl 2-0, and a bolster dressing was applied to reduce dead space and seroma. For subpectoral pockets, after dissecting the pectoralis major, an implant was inserted beneath the posterior surface of the muscle. After insertion, the pectoralis major flap was extended inferolaterally, and the inferior pole 
of the breast was extended using ADMs. Textured implants from Allergan, Mentor, or Polytech were used. Regardless of the brand, the implant was selected based on the parenchymal weight of the affected breast, preoperative measurements of both breasts (such as height, width, projection, and suprasternal notch to nipple distance), and symmetry and shape in relation to the contralateral breast. Four types of ADM (AlloDerm, LifeCell Corp., The Woodlands, TX, USA; CG CryoDerm, CGBio Co., Seongnam, Korea; MegaDerm, L\&C Bio Corp., Seoul, Korea; SurgiMend, Integra LifeSciences Corp., Plainsboro, NJ, USA) were used, in which $0.5-\mathrm{cm}$-diameter drain holes at $1-\mathrm{cm}$ intervals were made using a no. 11 blade before use. Every member of the surgical team changed surgical gloves before touching with implants and ADMs to reduce infection, and surgical instruments were disinfected once again with $80 \%$ ethanol. The pockets were irrigated with povidone-iodine and Adam's solution containing Tazocin (piperacillin and tazobactam), and subsequently, implants sterilized with povidone-iodine were inserted. Negative-pressure drainage $(400 \mathrm{~mL})$ was applied, and the drain was removed when the amount of drainage was $<30 \mathrm{~mL}$ for 2 consecutive days.

\section{Diagnosis of infection}

Infection was diagnosed based on clinical findings and results of physical examination and laboratory tests, including culture. In cases of suspected seroma, ultrasonography (US) was additionally performed. In cases of skin necrosis or wound discharge, culture was conducted directly from the wound. If there was a fluid collection (e.g., seroma) in the pocket, a specimen was collected aseptically through US-guided aspiration and cultured. The differential diagnosis included red breast syndrome, toxic shock syndrome, and sepsis.

Infections with symptoms of heat, edema, and cellulitis without drainage that were sensitive to initial antibiotic therapy were considered to be mild. A diagnosis of severe infection was rendered for patients with persistent edema despite antibiotic therapy, purulent drainage, or one or more symptoms of systemic infection and for those in which Pseudomonas, mycobacteria or Gram-negative rods were identified.

\section{Treatment of infection}

Implant salvage was defined as the maintenance of breast reconstruction after surgical treatment, including implant replacement [10]. By definition, salvage treatment involved invasive therapy; in other words, simple, noninvasive antibiotic treatment was not counted as salvage treatment. Early aggressive salvage treatment was defined as invasive treatment within 1 week after the infection.
Patients suspected of having an infection underwent antibiotic therapy, debridement of necrotic tissue (e.g., areas of post-mastectomy skin necrosis and the infected pocket), aspiration of seroma, incision and drainage (I\&D), massive antibiotic lavage in the implant pocket, and implant exchange. The management of patients suspected of having an infection is illustrated in Fig. 1. These patients were actively treated by multidisciplinary consultation and initially administered intravenous empirical antibiotics (Tazocin and vancomycin), followed by wound culture. After the identification of microorganisms through culture, the department of infectious diseases was consulted to switch to antibiotics that were sensitive to the specific microorganisms. Infected patients requiring salvage treatment underwent additional procedures such as debridement, povidone-iodine and antibiotic lavage in the implant pocket, and implant replacement. If there was no response to treatment, the implant was removed. If the amount of drainage did not decrease to $<30 \mathrm{~mL}$ for 2 consecutive days after 3 weeks, the drain tube was removed, as a possible source of infection, and US-guided aspiration was performed.

Whether the treatment resulted in improvement or exacerbation was determined based on clinical findings and the results of physical examination and laboratory tests. Patients' treatment response, future treatment planning, and infection control strategies were discussed at a biweekly breast team conference by specialists from the departments of plastic surgery, breast surgery, infectious diseases, and hemato-oncology. Salvage failure was defined as failure to maintain the implant pocket resulting from implant removal.

\section{Statistical analysis}

Statistical analysis was performed using SPSS version 22.0 (IBM Corp., Armonk, NY, USA). P-values $<0.05$ were considered to indicate statistical significance.

\section{RESULTS}

\section{Patient demographics}

A total of 145 cases of implant-based breast reconstruction, including 13 cases of bilateral reconstruction, were performed in 132 patients between January 2012 and December 2018. The demographic characteristics of the patients are summarized in Table 1. Three of the five patients with bilateral breast cancer underwent bilateral implant-based breast reconstruction, and 10 of the 127 patients with unilateral breast cancer underwent contralateral breast augmentation along with ipsilateral breast reconstruction.

In the patients who did not develop infections, the mean age 


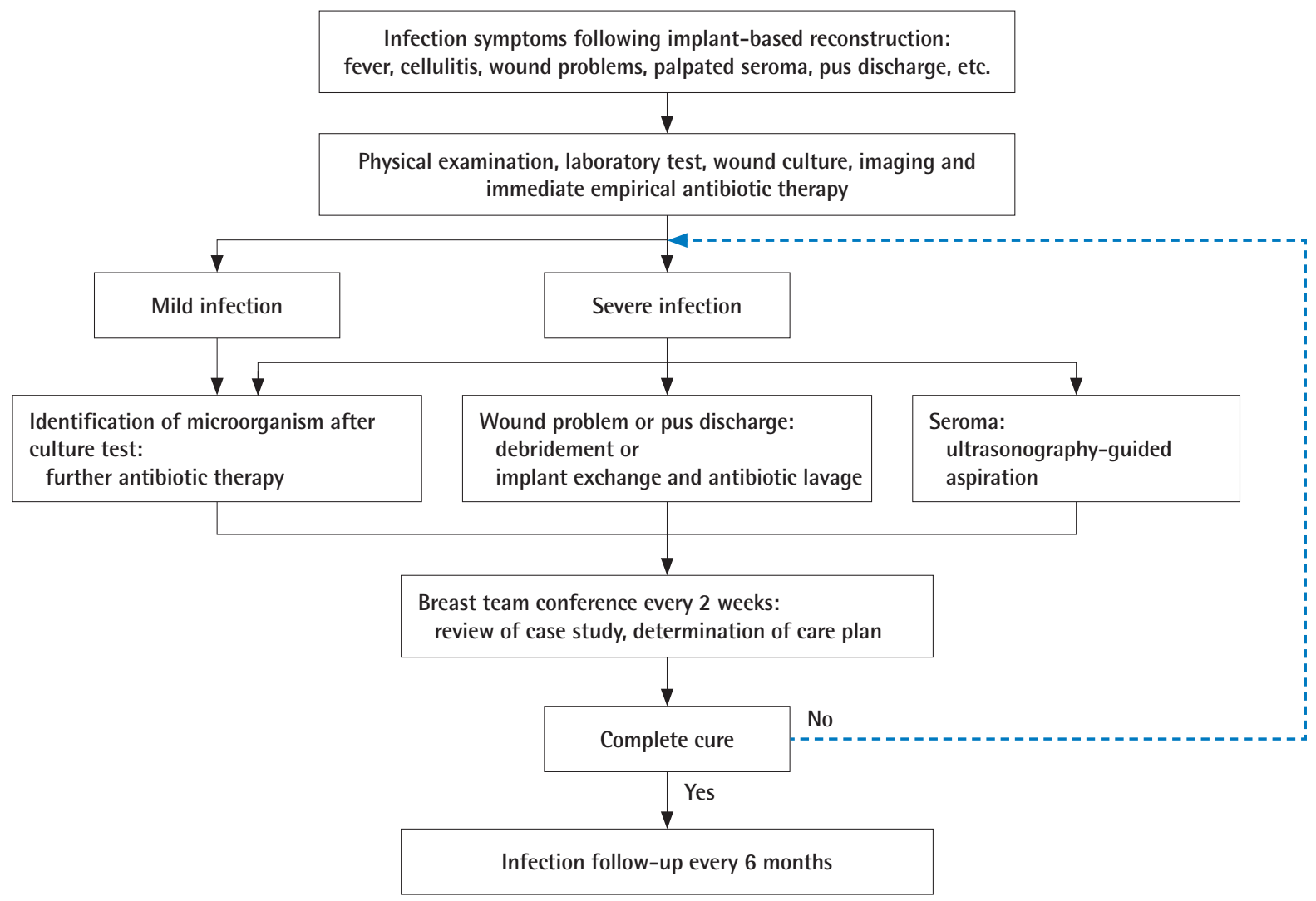

Fig. 1. Management of patients with infections.

Table 1. Demographic characteristics of the patients

\begin{tabular}{|c|c|c|c|}
\hline Characteristics & Patients with infections & Patients without infections & P-value \\
\hline No. of patients & 8 & 124 & \\
\hline No. of breasts & 8 & 137 & \\
\hline Age (yr) & $48.50(34-66)$ & $45.18(18-68)$ & 0.296 \\
\hline BMl $\left(\mathrm{kg} / \mathrm{m}^{2}\right)$ & $22.28(17.10-28.58)$ & 21.89 (16.69-34.05) & 0.710 \\
\hline Smokers & 0 & 0 & - \\
\hline Hypertension & 1 & 32 & 0.400 \\
\hline Diabetes & 1 & 9 & 0.598 \\
\hline Period of indwelling catheter (day) & 18.6 & 12.4 & 0.008 \\
\hline Adjuvant Tx & 5 & 37 & 0.045 \\
\hline CTx & 2 & 20 & 0.349 \\
\hline RTx & 0 & 4 & 1.000 \\
\hline $\mathrm{CTx}+\mathrm{RT} x$ & 3 & 13 & 0.044 \\
\hline LND & 8 & 106 & 0.202 \\
\hline SLND & 7 & 98 & 0.445 \\
\hline ALND & 1 & 8 & 0.409 \\
\hline \multicolumn{4}{|l|}{ Pocket plane } \\
\hline Subpectoral & 8 & 104 & 0.199 \\
\hline Prepectoral & 0 & 33 & 0.199 \\
\hline \multicolumn{4}{|l|}{ Complication } \\
\hline Flap necrosis & 3 & 8 & 0.015 \\
\hline Hematoma & 1 & 5 & 0.293 \\
\hline Seroma & 4 & 16 & 0.013 \\
\hline Implant rupture & 1 & 0 & 0.055 \\
\hline Capsular contracture & 2 & 4 & 0.036 \\
\hline
\end{tabular}

BMI, body mass index; Tx, treatment; CTx, chemotherapy; RTx, radiation therapy; LND, lymph node dissection; SLND, sentinel lymph node dissection; ALND, axillary lymph node dissection. 
was 45.18 years (range, 18-68 years), and the mean BMI was $21.94 \mathrm{~kg} / \mathrm{m}^{2}$ (range, $16.69-34.05 \mathrm{~kg} / \mathrm{m}^{2}$ ), whereas in patients who developed an infection, the mean age was 46.5 years (range, 34-66 years), and the mean BMI was $21.70 \mathrm{~kg} / \mathrm{m}^{2}$ (range, $17.10-28.58 \mathrm{~kg} / \mathrm{m}^{2}$ ); neither of these differences were statistically significant. Sixteen patients had a history of concurrent chemoradiotherapy, and four and 22 patients had received radiotherapy or chemotherapy alone, respectively. Of the eight patients with infections, three had received concurrent chemoradiotherapy and two had received chemotherapy alone. A total of 114 patients - including all eight patients who developed infections - underwent axillary lymph node dissection, including axillary sentinel lymph node dissection.

There was no significant association between axillary lymph node dissection and infection $(\mathrm{P}=0.202)$, whereas significant associations were observed for adjuvant therapy $(\mathrm{P}=0.045)$ and concurrent chemoradiotherapy $(\mathrm{P}=0.044)$.

Of the 145 cases of implant-based breast reconstruction, the implant was inserted into a subpectoral pocket in 104 cases and into a prepectoral pocket in 33 cases. All eight infections occurred in the subpectoral pocket group. The mean duration of negative-pressure drainage was 12.4 days in patients who did not develop infections and 18.6 days in patients who developed infections $(\mathrm{P}=0.008)$.

\section{Diagnosis and treatment}

The demographic data of the patients who developed infections are shown in Table 2 . The overall rate of infection was $5.5 \%$ $(8 / 145)$, and the salvage rate in patients with infections was $87.5 \%(7 / 8)$. The characteristics and treatment modalities for patients with infections are presented in Table 3 . These patients underwent antibiotic therapy, debridement of necrotic tissue, aspiration, I\&D, antibiotic lavage in the implant pocket, and implant exchange. The ADMs were retained when the implants were replaced. All patients with infections in this study had severe infections. One patient with a methicillin-resistant Staphy-

Table 2. Demographic characteristics of the patients with infections

\begin{tabular}{lcccccccc}
\hline Patient & Age $(\mathrm{yr})$ & $\begin{array}{c}\mathrm{BMl} \\
\left(\mathrm{kg} / \mathrm{m}^{2}\right)\end{array}$ & $\begin{array}{c}\text { Underlying } \\
\text { disease }\end{array}$ & $\begin{array}{c}\text { Chemo } \\
\text { therapy }\end{array}$ & $\begin{array}{c}\text { Radiation } \\
\text { therapy }\end{array}$ & $\begin{array}{c}\text { Axillary node } \\
\text { dissection }\end{array}$ & $\begin{array}{c}\text { Use of ADM } \\
\text { (day) }\end{array}$ \\
\hline 1 & 40 & 17.10 & Gastric cancer & + & + & SLNB & AlloDerm \\
2 & 52 & 20.13 & HBV & - & - & SLNB & AlloDerm & 21 \\
3 & 45 & 21.49 & - & + & + & ALND & CGCryoDerm & 17 \\
4 & 66 & 23.80 & DM & + & + & SLNB & CGCryoDerm & 19 \\
5 & 51 & 23.28 & - & + & - & SLNB & SurgiMend & 14 \\
6 & 47 & 24.17 & - & - & - & SLNB & MegaDerm & 12 \\
7 & 53 & 19.71 & - & - & - & SLNB & SurgiMend & 17 \\
8 & 34 & 28.58 & HTN & + & - & SLNB & SurgiMend & 26 \\
\hline
\end{tabular}

BMI, body mass index; ADM, acellular dermal matrix; SLNB, sentinel lymph node biopsy; ALND, axillary lymph node dissection; HBV, hepatitis B virus infection; DM, diabetes mellitus; HTN, hypertension.

Table 3. Features and management of the patients with infections

\begin{tabular}{|c|c|c|c|c|c|c|c|c|c|}
\hline Patient & $\begin{array}{l}\text { Onset of } \\
\text { signs of } \\
\text { infection } \\
\text { (POD) }\end{array}$ & $\begin{array}{l}\text { Date of starting } \\
\text { antibiotic } \\
\text { treatment } \\
\text { (POD) }\end{array}$ & $\begin{array}{l}\text { Date of } \\
\text { surgical } \\
\text { intervention } \\
\text { (POD) }\end{array}$ & $\begin{array}{l}\text { Infection } \\
\text { type }\end{array}$ & $\begin{array}{l}\text { Date of } \\
\text { microorganism } \\
\text { Identification } \\
\text { (POD) }\end{array}$ & $\begin{array}{l}\text { Microorganism } \\
\text { Identified }\end{array}$ & $\begin{array}{l}\text { Antibiotic } \\
\text { change }\end{array}$ & Treatment & $\begin{array}{l}\text { Implant } \\
\text { exchange } \\
\text { (POD) }\end{array}$ \\
\hline 1 & 9 & 10 & 12 & Severe & 15 & $P$. aeruginosa & Ciprofloxacin & $\begin{array}{l}\text { Antibiotic therapy, antibiotic } \\
\text { lavage, implant exchange }\end{array}$ & 12 \\
\hline 2 & 15 & 17 & 17 & Severe & 22 & P. aeruginosa & Tazocin & $\begin{array}{l}\text { Antibiotic therapy, antibiotic } \\
\text { lavage, implant exchange }\end{array}$ & 17 \\
\hline 3 & 39 & 39 & 42 & Severe & 42 & P. aeruginosa & Ciprofloxacin & $\begin{array}{l}\text { Antibiotic therapy, antibiotic } \\
\text { lavage, implant exchange }\end{array}$ & 42 \\
\hline 4 & 5 & 0 & 8 & Severe & 8 & $\begin{array}{l}\text { Acinetobacter } \\
\text { baumannii }\end{array}$ & Meropenem & $\begin{array}{l}\text { Antibiotic therapy, debridement, } \\
\text { aspiration, implant exchange }\end{array}$ & 8 \\
\hline 5 & 25 & 25 & 26 & Severe & 30 & No growth & Vancomycin & $\begin{array}{l}\text { Antibiotic therapy, aspiration, } \\
\text { implant exchange }\end{array}$ & 26 \\
\hline 6 & 11 & 12 & 16 & Severe & 16 & MRSA & Meropenem & $\begin{array}{l}\text { Antibiotic therapy, debridement, } \\
\text { aspiration, implant exchange }\end{array}$ & 16 \\
\hline 7 & 47 & 47 & 47 & Severe & 52 & MRSE & Vancomycin & $\begin{array}{l}\text { Antibiotic therapy, aspiration, } \\
\text { implant exchange }\end{array}$ & 47 \\
\hline 8 & 32 & 43 & 43 & Severe & 48 & MRSA & Meropenem & Implant removal (salvage failure) & 43 \\
\hline
\end{tabular}

POD, postoperative day; P. aeruginosa, Pseudomonas aeruginosa; MRSA, methicillin-resistant Staphylococcus aureus; MRSE, methicillin-resistant Staphylococcus epidermidis. 
lococcus aureus (MRSA) infection showed no response to systemic antibiotic therapy, drainage, antibiotic lavage, hematoma and necrotic tissue removal, and implant replacement. Therefore, explantation was performed, and this case was regarded as an instance of salvage failure. The other seven patients were aggressively treated within 1 week, and salvage treatment succeeded in all seven.

Culture tests demonstrated Pseudomonas aeruginosa, which had the highest frequency, in three patients, MRSA in two, and methicillin-resistant Staphylococcus epidermidis (MRSE) in one. The mean follow-up period after complete recovery from infection was 13.2 months. When infection was suspected, Tazocin and vancomycin were used as the initial empirical intravenous antibiotics until the identification of microorganisms based on culture. After the microorganisms were identified, other antibiotics were used according to antibiotic sensitivity. For $P$. aeruginosa, the antibiotic regimen was changed to Tazocin or ciprofloxacin. When MRSA or MRSE was identified, vancomycin was used. Banan (cefpodoxime proxetil) and Unasyn (sultamicillin tosylate) were used as oral medications. The average duration of intravenous antibiotic use was 24 days (interquartile range, 11-29 days) and the average duration of oral antibiotic use was 10.875 days (interquartile range, $7-14$ days).

\section{Complications}

The complications are shown in Table 1 and Fig. 2. Seroma, which was defined as persistent seroma over 3 weeks requiring aspiration, was the most common complication (20/145, $13.8 \%)$, followed by wound-related complications such as skin necrosis or wound dehiscence $(11 / 145,7.6 \%)$. In the patients who developed infections, seroma $(4 / 8,50.0 \%)$ was the most common complication, followed by wound-related complications $(3 / 8,37.5 \%)$, capsular contracture $(2 / 8,25.0 \%)$, implant rupture $(1 / 8,12.5 \%)$, and hematoma $(1 / 8,12.5 \%)$. After sal- vage, no complications were identified other than capsular contracture $(2 / 8,25.0 \%)$. No significant differences were found in the occurrence of hematoma $(\mathrm{P}=0.293)$ or implant rupture $(\mathrm{P}=0.055)$ according to whether patients developed an infection. However, significant between-group differences were found in the occurrence of flap necrosis $(P=0.015)$, seroma $(\mathrm{P}=0.013)$, and capsular contracture $(\mathrm{P}=0.036)$.

\section{Case presentation}

Case 1: salvage through initial treatment of infection (antibiotic therapy, antibiotic lavage, implant exchange)

A 40-year-old woman with a history of partial gastrectomy for gastric cancer and breast augmentation using 180-cc silicone implants presented with a left breast mass. After the diagnosis of breast cancer, she underwent mastectomy (mass, $142 \mathrm{~g}$ ) at the department of general surgery. The previous implant was removed, and a 253-cc textured, round silicone implant from Allergan was inserted subpectorally. AlloDerm (LifeCell Corp.) with an area of $4 \times 16 \mathrm{~cm}$ and 0.53 - to $0.76-\mathrm{mm}$ thickness was used. The drainage tube was maintained for 21 days after surgery. The patient underwent radiotherapy and chemotherapy. Although the patient showed good progress until 8 days after surgery, on day 9 there was an increase in the erythematous area with local heat, tenderness, and fever (body temperature, $38.4^{\circ} \mathrm{C}$ ). A blood test showed an elevated C-reactive protein (CRP) level $(25.88 \mathrm{mg} / \mathrm{L})$ and white blood cell count $(21,450$ cells $/ \mu \mathrm{L})$. The patient was referred to the Departments of Breast Surgery and Infectious Diseases and was intravenously administered empirical broad-spectrum antibiotics (Tazocin and vancomycin). Because no improvement was observed by day 3 of salvage treatment, the patient underwent antibiotic lavage, debridement of necrotic tissue, and implant substitution. A culture test performed on the intraoperatively obtained tissue showed P. aeruginosa, and accordingly, the antibiotics were changed to ciprofloxacin



Fig. 2. Complications of the patients. 
and rifampicin after consultation with the department of infectious diseases. After salvage, all clinical findings and laboratory test results improved, and the patient was discharged without complications on day 25 . No complications such as capsular contracture or implant infection were found during 6 months of follow-up after salvage (Fig. 3).

\section{Case 2: salvage failure}

A 34-year-old woman underwent mastectomy for left breast cancer and breast reconstruction using a 470-cc textured, anatomic silicone implant from Allergan. The implant was inserted
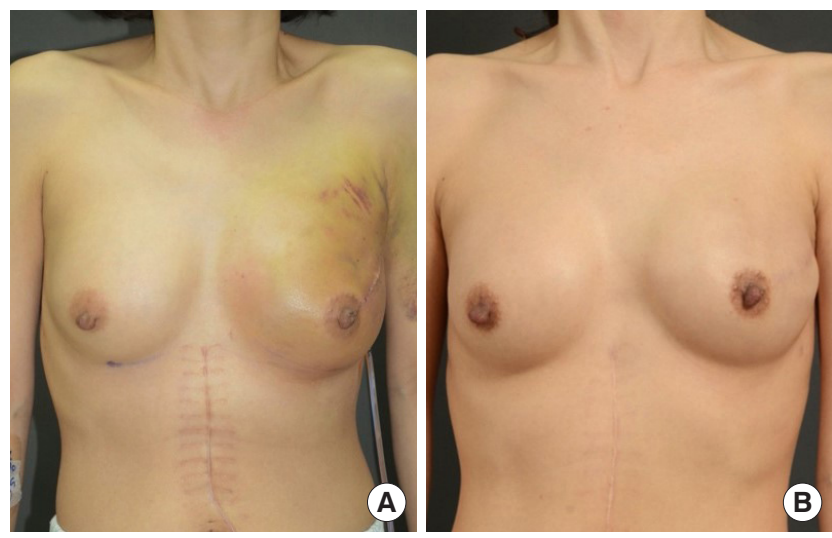

Fig. 3. Case of successful salvage through initial treatment. A 40-year-old woman with ductal carcinoma in situ in the left breast. (A) Generalized erythema and swelling on the left breast with tenderness before salvage. (B) Recovery without complications 6 months after salvage. subpectorally, and SurgiMend 1.0 (Integra LifeSciences Corp.) with an area of $6 \times 16 \mathrm{~cm}$ and 1-mm thickness was used. The drainage tube was maintained for 26 days after surgery. The patient underwent chemotherapy. On day 32 , swelling and local heat occurred in the left breast, and the patient was admitted 43 days after the onset of symptoms. A blood test showed a CRP level of $5.58 \mathrm{mg} / \mathrm{L}$ and a white blood cell count of 7,730 cells/ $\mu \mathrm{L}$. Broad-spectrum systemic antibiotics were administered immediately, and consultations with the department of breast surgery, infectious diseases, and radiology were concurrently conducted. The patient did not respond to antibiotic therapy, and seroma with infectious findings was observed on radiography, including US and computed tomography. Salvage was performed in the following order: aspiration of seroma, antibiotic lavage, hematoma and necrotic tissue removal, and implant replacement. However, the patient's symptoms did not improve, and the implant was eventually removed after a breast team conference (Fig. 4).

\section{DISCUSSION}

The infection rate of implants in breast reconstruction patients ranges from $1 \%$ to $35 \%$, and implant exposure occurs in up to $8 \%$ of cases. Implant infections are traditionally treated by infection control through implant removal and antibiotic therapy, after which reconstruction is performed [13-15]. However, since Courtiss et al. [16] reported a study on the salvage procedure for implant infection after breast augmentation using implants in 1965, multiple reports have described the efficacy of early sal-
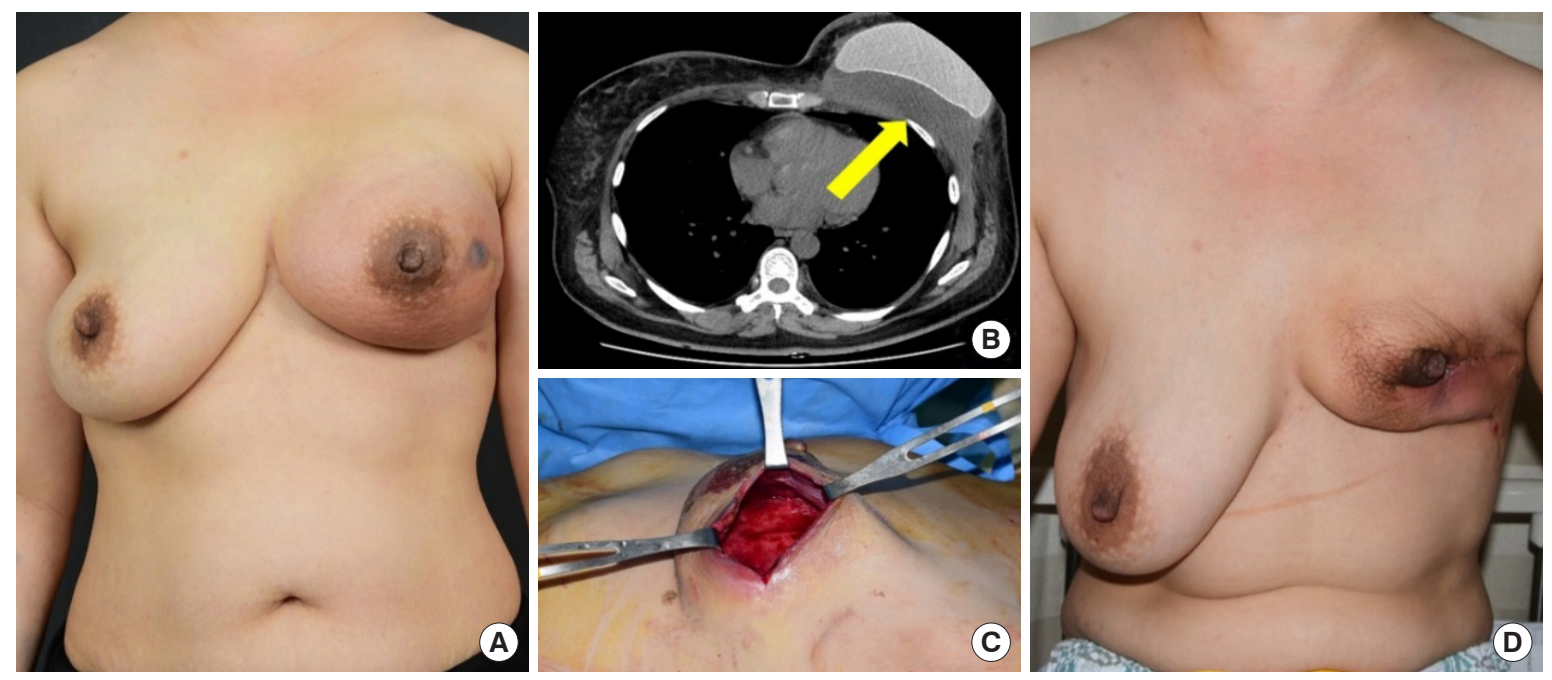

Fig. 4. Case of salvage failure. A 34-year-old woman with invasive ductal carcinoma in the left breast. (A) Generalized erythema and swelling of the left breast with tenderness and some skin flap necrosis before salvage. (B) Seroma and hematoma on radiological examination (yellow arrow). (C, D) Explantation due to salvage failure despite antibiotic lavage and implant exchange. 
vage for implant infection $[10,11,17]$. According to Chun et al. in 2007 [10], eight patients with infections received systemic antibiotics along with a surgical intervention for cyst enucleation and implant replacement, and were salvaged without reinfection. Moreover, no capsular contracture was observed in the replaced implants. In 2012, Prince et al. [11] conducted a retrospective study on 60 patients who underwent secondary surgical treatment due to infection or exposure of breast implants between 2002 and 2008 and reported that $76.7 \%$ of cases showed successful salvage. The authors insisted that it was essential not only to perform a rapid and active surgical intervention, but also to administer systemic antibiotic therapy, and proposed a simplified protocol to maximize the salvage rate in patients with infection and exposure of breast implants. In 2013, Reish et al. [17] conducted a retrospective study on 1,241 patients with postoperative infections after breast reconstruction between 2004 and 2010, and reported an approximately $37.3 \%$ salvage rate by systemic antibiotic therapy and implant replacement.

Some studies have suggested that a multidisciplinary approach maximizes the efficacy of infection treatment. Trop et al. [18] reported that a multidisciplinary team approach was more effective for the treatment of breast abscesses than traditional I\&D by surgeons. Schlarb et al. [19] reported successful treatment using an interdisciplinary approach for mycobacterial infection after abdominoplasty, which can lead to serious complications.

Based on a study by Spear and Seruya [15], infections were classified as mild or severe in the present study. Mild infection was defined as warmth, swelling, or cellulitis without drainage that was responsive to initial antibiotic therapy, whereas severe infection was defined as any case that met one or more of the following criteria: persistent and substantial swelling despite antibiotic therapy, purulent drainage with or without cellulitis, aggressive or atypical organisms on culture (e.g., Pseudomonas, mycobacteria, or Gram-negative rods), or serious signs and symptoms of systemic infection (e.g., hypotension or high fever). All of the infections identified in this study were severe, and active treatment was performed immediately after the identification of suspicious signs or symptoms. Despite having severe infections, seven patients could be salvaged by early aggressive treatment within 1 week.

In this study, after the first occurrence of signs of infection such as fever, pain at the surgical site, and elevated CRP levels, systemic antibiotic therapy was immediately initiated, and rapid diagnosis and treatment were performed through an active multidisciplinary consultation. When needed, salvage therapy was performed by aseptic aspiration of seroma, debridement of necrotic tissue, antibiotic lavage, and implant replacement, and patients were discharged without complications at an average of
9.7 days after salvage. Of the eight patients with infections, the total salvage rate was $87.5 \%$, and all patients who were salvaged by early aggressive treatment within 1 week showed successful results. No complications or reinfections, except capsular contracture in two patients, were observed after salvage during a mean follow-up period of 13.2 months.

In this study, we replaced the infected breast implant with a new one, rather than simply washing or soaking it. In 2010, Feldman et al. [20] reported that when a biofilm is formed by bacterial adherence to a breast implant, the bacteria are resistant to antibiotics to which they are susceptible while in suspension. Furthermore, vancomycin, one of the antibiotics that we used, has decreased activity against MRSA in a biofilm. In 2007, Chun et al. [10] reported that implant exchange was the critical factor in salvage. Simply washing or soaking an infected implant may not remove the bacteria properly, while replacing it with a new implant provides an opportunity to prevent the recurrence of infection. Based on the above study, we performed implant exchange in patients with infections.

One of the reasons for salvage failure is the suboptimal treatment of MRSA infection. According to a report by Song et al. [21], MRSA is the most commonly identified strain in implantrelated infections and an important factor for explantation. Yii and Khoo [22] confirmed that the salvage rate was lower in breast reconstructions with MRSA infection. A study of 69 patients with infected or exposed breast implants by Spear and Seruya in 2010 reported that infection with Gram-negative rods, MRSA, and Candida parapsilosis was associated with a higher probability of salvage failure $[15,21]$. The infection sources could include community-acquired pathogens and contamination from medical staff who participated in the operation. Considering the characteristics of breast surgery, a relatively large number of medical staff from multiple departments, including breast surgery and plastic surgery, participate in surgery, thereby increasing the possibility of exposure to refractory infectious strains. In this study, Flumarin (flomoxef) was used as a prophylactic antibiotic, and some bacteria that are not covered by Flumarin may have caused infection with antibiotic-resistant bacteria. Another possible contributor to salvage failure is delayed admission to the hospital despite symptoms of postoperative infection. In addition, the lack of surgical techniques to reduce seroma at the early stage of study could have been a reason for salvage failure.

The superior salvage rate of the present study cannot be directly compared with other studies because of inconsistencies across studies in the definitions of infection in implant-based breast reconstruction, which might correspond to differences in the selection of cases for salvage treatment. There are also dis- 
agreements in the interpretation of salvage results and bias due to differences in the number of surgical staff. In addition, this study was conducted as a retrospective chart review, and therefore it was not possible to propose a systematic and clear algorithm for the diagnosis and treatment of infection.

Interestingly, no additional cases of infection requiring salvage occurred at our center in patients with implant-based breast reconstruction, as regular breast team conferences have been held to prevent infection and seroma and intraoperative techniques, with appropriate consultations, have been applied to reduce seroma through active aspiration. Despite its several limitations, this study demonstrates that aggressive salvage treatment within 1 week is essential in cases of peri-implant infection. For salvage treatment, empirical systemic antibiotic therapy should be performed immediately after the identification of a suspected infection, and rapid diagnosis and treatment planning must take place through a multidisciplinary consultation, which can facilitate proper antibiotic therapy and active surgical intervention.

\section{NOTES}

\section{Conflict of interest}

No potential conflict of interest relevant to this article was reported.

\section{Ethical approval}

The study was approved by the Institutional Review Board of Kyungpook National University Chilgok Hospital (IRB No. KNUCH 2020-07-019) and performed in accordance with the principles of the Declaration of Helsinki. Written informed consent was obtained.

\section{Patient consent}

The patients provided written informed consent for the publication and the use of their images.

\section{Author contribution}

Conceptualization: H Yeo, JS Kim, JS Lee, JD Yang. Data curation, formal analysis: D Lee, PS Eo. Methodology: H Yeo, JD Yang. Project administration: JD Yang. Visualization: D Lee, JS Kim, JD Yang. Writing - original draft: H Yeo. Writing - review \& editing: D Lee, JS Kim, PS Eo, DK Kim, JS Lee, KT Kwon, J Lee, HY Park, JD Yang.

\section{ORCID}

Hyeonjung Yeo

https://orcid.org/0000-0001-5705-7380

Dongkyu Lee https://orcid.org/0000-0003-3395-3775

Jin Soo Kim
Pil Seon Eo

Dong Kyu Kim

Joon Seok Lee

Ki Tae Kwon

Jeeyeon Lee

Ho Yong Park

Jung Dug Yang https://orcid.org/0000-0003-1909-7298

https://orcid.org/0000-0003-1679-8654

https://orcid.org/0000-0002-1580-0487

https://orcid.org/0000-0003-4666-0672

https://orcid.org/0000-0003-1826-1690

https://orcid.org/0000-0003-2318-9806

https://orcid.org/0000-0002-9040-4724

\section{REFERENCES}

1. American Society of Plastic Surgeons (ASPS). 2018 Plastic surgery statistics report [Internet]. Arlington Heights, IL: ASPS; c2019 [cited 2021 Mar 9]. Available from: Available at: https://www.plasticsurgery.org/documents/News/ Statistics/2018/plastic-surgery-statistics-full-report-2018. pdf.

2. Brown MH, Shenker R, Silver SA. Cohesive silicone gel breast implants in aesthetic and reconstructive breast surgery. Plast Reconstr Surg 2005;116:768-79.

3. Kim JB, Jeon HJ, Lee JW, et al. A murine model of radiationinduced capsule-tissue reactions around smooth silicone implants. J Plast Surg Hand Surg 2018;52:217-24.

4. Araco A, Gravante G, Araco F, et al. A retrospective analysis of 3,000 primary aesthetic breast augmentations: postoperative complications and associated factors. Aesthetic Plast Surg 2007;31:532-9.

5. Sullivan SR, Fletcher DR, Isom CD, et al. True incidence of all complications following immediate and delayed breast reconstruction. Plast Reconstr Surg 2008;122:19-28.

6. Cordeiro PG, McCarthy CM. A single surgeon's 12-year experience with tissue expander/implant breast reconstruction: part I. a prospective analysis of early complications. Plast Reconstr Surg 2006;118:825-31.

7. De Cholnoky T. Augmentation mammaplasty: survey of complications in 10,941 patients by 265 surgeons. Plast Reconstr Surg 1970;45:573-7.

8. Warren Peled A, Itakura K, Foster RD, et al. Impact of chemotherapy on postoperative complications after mastectomy and immediate breast reconstruction. Arch Surg 2010; 145:880-5.

9. Leyngold MM, Stutman RL, Khiabani KT, et al. Contributing variables to post mastectomy tissue expander infection. Breast J 2012;18:351-6.

10. Chun JK, Schulman MR. The infected breast prosthesis after mastectomy reconstruction: successful salvage of nine implants in eight consecutive patients. Plast Reconstr Surg 2007;120:581-9.

11. Prince MD, Suber JS, Aya-Ay ML, et al. Prosthesis salvage in 
breast reconstruction patients with periprosthetic infection and exposure. Plast Reconstr Surg 2012;129:42-8.

12. Lee JS, Kim JS, Lee JH, et al. Prepectoral breast reconstruction with complete implant coverage using double-crossed acellular dermal matrixs. Gland Surg 2019;8:748-57.

13. Alderman AK, Wilkins EG, Kim HM, et al. Complications in postmastectomy breast reconstruction: two-year results of the Michigan Breast Reconstruction Outcome Study. Plast Reconstr Surg 2002;109:2265-74.

14. Gabriel SE, Woods JE, O’Fallon WM, et al. Complications leading to surgery after breast implantation. N Engl J Med 1997;336:677-82.

15. Spear SL, Seruya M. Management of the infected or exposed breast prosthesis: a single surgeon's 15-year experience with 69 patients. Plast Reconstr Surg 2010;125:1074-84.

16. Courtiss EH, Goldwyn RM, Anastasi GW. The fate of breast implants with infections around them. Plast Reconstr Surg 1979;63:812-6.

17. Reish RG, Damjanovic B, Austen WG Jr, et al. Infection fol- lowing implant-based reconstruction in 1952 consecutive breast reconstructions: salvage rates and predictors of success. Plast Reconstr Surg 2013;131:1223-30.

18. Trop I, Dugas A, David J, et al. Breast abscesses: evidencebased algorithms for diagnosis, management, and follow-up. Radiographics 2011;31:1683-99.

19. Schlarb D, Idelevich EA, Krause-Bergmann A, et al. Successful interdisciplinary radical treatment of Mycobacterium fortuitum infection in a lipotourist from Germany after abdominoplasty in Turkey. New Microbes New Infect 2015;8: 21-3.

20. Feldman EM, Kontoyiannis DP, Sharabi SE, et al. Breast implant infections: is cefazolin enough? Plast Reconstr Surg 2010;126:779-85.

21. Song JH, Kim YS, Jung BK, et al. Salvage of infected breast implants. Arch Plast Surg 2017;44:516-22.

22. Yii NW, Khoo CT. Salvage of infected expander prostheses in breast reconstruction. Plast Reconstr Surg 2003; 111: 1087-92. 